\title{
Research Idea on How Language and Symbols (Semantics and Semiotics) Affect Emotions of Software Engineers
}

\author{
Mahesh Venkataraman \\ Quality Engineering, Accenture \\ India \\ mahesh.venkata.raman@accenture.com
}

\author{
Kishore Durg \\ Intelligent Cloud \& Infrastructure, Accenture \\ India \\ kishore.p.durg@accenture.com
}

\begin{abstract}
This is essentially a 'call for research' and collaboration between industry and academia to improve the motivation and performance of software engineers through use of language, words and symbols.

How languages and symbols shape the way people think, feel and behave has been a topic of wide research. Words have powerful association with perception and cognition and throughout history, language has been used as a medium for influencing minds and for mass propaganda. While this is widely understood in politics, psychology and sociology, very little research has been to study the implicit and explicit impact of words, phrases and language on the way software engineers think, feel, behave and perform. While software engineering could be seen as a science that lends itself to a formal process and methods, it can also be seen as a craft and art which needs imagination and creativity which in turn are influenced by emotions. We propose some hypotheses, research questions and ideas to trigger formal studies of deeper connections between language/ symbols and software engineers' performance. We also draw inspiration from a wide body of research already conducted in this area which have influenced the field of psychology, sociology and mass communication. This is essentially a 'call for research' and collaboration between industry and academia to improve the motivation and performance of software engineers through use of language, words and symbols.
\end{abstract}

\section{KEYWORDS}

emotions, language, performance, software engineering, symbols, semiotics

\section{ACM Reference Format:}

Mahesh Venkataraman and Kishore Durg. 2020. Research Idea on How Language and Symbols (Semantics and Semiotics) Affect Emotions of Software Engineers. In IEEE/ACM 42nd International Conference on Software Engineering Workshops (ICSEW'20), May 23-29, 2020, Seoul, Republic of Korea. ACM, New York, NY, USA, 4 pages. https://doi.org/10.1145/3387940.3392232

\section{OBJECTIVE}

The objective of this short paper is to propose industry-academic collaborative research on how language and symbols (semantics

Permission to make digital or hard copies of part or all of this work for personal or classroom use is granted without fee provided that copies are not made or distributed for profit or commercial advantage and that copies bear this notice and the full citation on the first page. Copyrights for third-party components of this work must be honored

For all other uses, contact the owner/author(s).

ICSEW'20, May 23-29, 2020, Seoul, Republic of Korea

(c) 2020 Copyright held by the owner/author(s).

ACM ISBN 978-1-4503-7963-2/20/05.

https://doi.org/10.1145/3387940.3392232 and semiotics) affect the way Software Engineers think, feel, behave and perform

\section{INTRODUCTION}

It has been established through research that language influences culture and conversely a culture is embedded in its languages. Language is so fundamental to our experience, so much embedded in our human existence, that it is hard to imagine life without it. At every stage in software development, imagination, creativity and mental visualization are involved and so the outputs of a software engineer are influenced by emotions. And research indicates emotions are influenced by words and symbols. Emotions also affect perception and cognition. In this paper we draw inspiration from a wide body of research done on how language (semantics) and symbology (semiotics) shape thinking, emotion and behaviour and pose some research questions on how a similar research could be conducted to empirically study the connection between language, symbols and performance of software engineers. Study of such influence also helps us in understanding how right terminology can affect emotions of software engineers leading to positive performance outcomes.

This proposed research can also include how people from various non-English speaking cultures emotionally respond differently to various English terms, phrases and jargons

The term 'language' in this paper includes the following:

- Vocabulary, terminology and jargons.

- Symbols and colours that signify a 'thing', status or call for action.

- Phraseology, patterns of repeated phrases and sentences to convey specific meaning.

- Specific terms, jargons and symbols used in software industry in general and specific team in particular.

- Language and symbols used in interactions between project stakeholders and software engineers.

- Language and symbols used in interactions between managers and team members.

- The lexicon used in the day to day interactions.

- Language and symbols used to describe project plans, status and outcomes.

- Language and symbols used in software artefacts and deliverables.

- The language and symbols used to measure progress and metrics.

- Terminology used to measure performance. 
We believe that the output of this (proposed) research could be used to understand how language, words and terminology and symbols can influence thinking, emotions, behaviour and performance of software engineers

\section{EXISTING RESEARCH}

We list below some relevant and prior research on how language and symbols shapes thinking, culture and behavior. Our proposed research will draw inspiration from these prior research work.

\subsection{Sapir-Whorf Hypothesis or the hypothesis of linguistic relativity}

This is a principle claiming that the structure of a language affects its speakers' world view or cognition, and thus people's perceptions are relative to their spoken language. Here again there are two variants; the first is linguistic determinism, which holds that language entirely determines the range of cognitive processes and the second is linguistic influence proposing that language provides constraints in some areas of cognition, but that it is by no means determinative. Research on weaker forms has produced positive empirical evidence for a relationship [5], [3].

\subsection{Linkage between language and emotion}

Research has proven strong correlation between language and emotion. According to the psychological constructionist Conceptual Act Theory (CAT), an emotional instance arises during an information transfer. And these emotions are highly contextual. This could be used to further conduct research on how phrases, words, language and symbols affect emotions of software engineers [4].

\subsection{Study on how language infuences future oriented behavior}

Research has established a link between language and future oriented behaviour [1]. We postulate that a person who is inherently future oriented has higher level of emotional stability. This also may reflect in the way the software engineer thinks, models, designs and codes in a way that is future proof.

\subsection{Concept of hypocognition}

In cognitive linguistics, hypocognition means missing and being unable to communicate cognitive and linguistic representations because there are no words for particular concepts. [7] George Orwell's 1984 featured a fictitious language called Newspeak of restricted grammar and limited vocabulary, meant to limit the freedom of thought-personal identity, self-expression, free will-that threatens the ideology of the régime. It would be worthwhile doing research on how positive or negative 'syndromes' or patterns of problems in software engineering are due to non-existent or ambiguous terms that defy adequate descriptions and interactions and thus increase the stress levels of software engineers thereby affecting their performance. Once understood, the industry could possibly coin new terms and phrases and symbols to adequately aid thinking, cognition and communication of these ideas

\subsection{Language as symbolic action}

Symbolic action is a term used by 20th-century rhetorician Kenneth Burke to refer to systems of communication that rely on symbols. In Language and Symbolic Action (1966), Burke states that all language is inherently persuasive because symbolic acts do something as well as say something. This book throws significant light on language and symbolism. Further research and empirical study are required to establish the connection between language and symbols and the trigger of emotions and action in the context of day in the life of software engineer.

\section{RESEARCH IDEA}

\subsection{Research questions}

We list down the research questions to motivate further empirical study of our hypothesis that language and symbols will affect the way Software Engineers think, feel, behave and perform. The research could be both qualitative and quantitative

(1) What is the correlation between creative outputs and emotion (example: joy) for a software engineer for various activities?

(2) What are the pathways to creative thinking (leading to high quality software) for a software engineer?

(3) How does the terminology currently being used in software engineering influence the emotion of software engineers? The terminology includes conversational phrases as well as technical terms.

(4) How does the specific terminology in the given projects influence the emotion of software engineers? The terminology includes conversational phrases as well as technical terms.

(5) What are the oft repeated patterns of phrases in software engineering industry and how do they affect emotions?

(6) What is the emotional response to scorecards, symbols and dashboards (dashboards have a lot of symbolic content)?

(7) How do language and symbols contribute to building software organization emotional culture in the longer term?

\subsection{Dimensions of stimuli and impact}

Robert Plutchik was professor emeritus at the Albert Einstein College of Medicine and adjunct professor at the University of South Florida. He proposed a psychoevolutionary classification approach for general emotional responses. [6]

He identified 8 primary emotions which are the basis for all others and are grouped into polar opposites:

(1) Joy and sadness

(2) Acceptance and disgust

(3) Fear and anger

(4) Surprise and anticipation

He also proposed the 'wheel of emotions' [6] which could be used to 'map' the impact of the stimuli.

For the proposed research we identify some dimensions of stimuli, wherein language and symbols possibly influence emotions and map these emotions using the 'Wheel of Emotion'. These dimensions are:

(1) Project management: status reports, dashboards, terminology and symbols. 
(2) Process: process definitions, terminology and symbols, meetings - phraseology and naming conventions.

(3) Measures and metrics: how metric naming conventions and their measurements impact emotions.

(4) Performance management: how performance measures and conversations can impact emotions.

The table below provides a summary overview of situations wherein the language and symbols used could have potential impact on emotions and performance and consequentially the end quality of software. The proposed research will include these situations (the situations given below are only indicative)

\subsection{Overview of research workflow}

The proposed research will study the impact of language and symbols (as defined in Introduction section) on cognition and emotion and finally on quality of software.

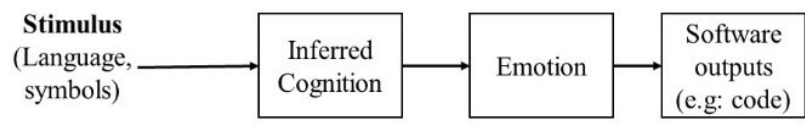

Figure 1: Research workflow

\subsection{Specific research methodology}

Since the author is from industry, this is best left to the academic community to decide!

\section{OTHER RELATED RESEARCH POSSIBILITIES}

\subsection{Artificial Languages and experimental semiotics [2]}

An artificial language is a language specially made for a purpose. These languages can be based on an existing natural language or can be artificial. We propose further research and study into creation of artificial languages that take into the unique thinking and motivation requirements of software engineers. These languages can be spoken in the context of a large software program to promote stress-free interactions and promote creative thinking and high-quality software

\subsection{How programming languages influence thinking and feelings [8]}

Another variant of this (again, proposed) research is to study how programming languages influence the way software engineers think, mentally model ideas, trigger creativity and evokes emotions of joy arising out of a creative pursuit thus impacting performance and quality of software

\section{ACKNOWLEDGMENTS}

Thanks to Janardan Misra for his assistance in the preparation of the paper.

\section{REFERENCES}

[1] M Keith Chen. 2013. The effect of language on economic behavior: Evidence from savings rates, health behaviors, and retirement assets. American Economic Review 103, 2 (2013), 690-731

[2] Bruno Galantucci. 2009. Experimental semiotics: A new approach for studying communication as a form of joint action. Topics in Cognitive Science 1, 2 (2009), 393-410.

[3] Paul Kay and Willett Kempton. 1984. What is the Sapir-Whorf hypothesis? American anthropologist 86, 1 (1984), 65-79.

[4] Kristen A Lindquist, Jennifer K MacCormack, and Holly Shablack. 2015. The role of language in emotion: Predictions from psychological constructionism. Frontiers in psychology 6 (2015), 444.

[5] John A Lucy. 1997. Linguistic relativity. Annual review of anthropology 26, 1 (1997), 291-312.

[6] Robert Plutchik and Henry Kellerman. 2013. Theories of emotion. Vol. 1. Academic Press.

[7] Kaidi Wu and David Dunning. 2018. Hypocognition: Making sense of the landscape beyond one's conceptual reach. Review of General Psychology 22, 1 (2018), 25-35.

[8] Jenna Zeigen. [n.d.]. Linguistic Relativity of Programming Languages. https://2014.jsconf.eu/speakers/jenna-zeigen-the-linguistic-relativity-ofprogramming-languages.html 


\begin{tabular}{|l|l|l|l|}
\hline \multirow{2}{*}{ Dimension } & \multicolumn{2}{|c|}{ Research possibilities - indicative only, not exhaustive } \\
\cline { 2 - 4 } & Situations & $\begin{array}{l}\text { Research questions } \\
\text { and symbols used in the meeting evoke? }\end{array}$ \\
\hline Project Management & Project vision meetings & $\begin{array}{l}\text { What kind of emotions did the language } \\
\text { and symbols used in the review evoke? }\end{array}$ & $\begin{array}{l}\text { Shared sense of progress, excitement, } \\
\text { fear }\end{array}$ \\
\hline Project Management & Project reviews & $\begin{array}{l}\text { What kind of emotions did the language } \\
\text { and symbols used in the process docu- } \\
\text { ment evoke? }\end{array}$ & $\begin{array}{l}\text { Sense of confidence of 'knowing', bore- } \\
\text { domse of wasteful effort, despair } \\
\text { due to bulk of process overheads }\end{array}$ \\
\hline Process & Process documents & $\begin{array}{l}\text { What kind of emotions did the language } \\
\text { and symbols used in the meeting evoke? }\end{array}$ & $\begin{array}{l}\text { Shared sense of responsibility, team ca- } \\
\text { maraderie and warmth, sense of envy } \\
\text { and jealousy }\end{array}$ \\
\hline Measures and metrics & $\begin{array}{l}\text { Metrics naming, status reports and dash- } \\
\text { boards }\end{array}$ & $\begin{array}{l}\text { What kind of emotions did the metrics } \\
\text { naming convention, status report lan- } \\
\text { guage, dashboard symbols have? }\end{array}$ & $\begin{array}{l}\text { Sense of shame/ pride/ nervousness/ } \\
\text { panic }\end{array}$ \\
\hline \hline
\end{tabular}

Table 1: Situation Table 